\title{
Sweet flavoured e-liquid
}

Addiction Ontology

\section{Source}

Addiction Ontology

Definition: A flavoured e-liquid in which the e-liquid flavouring is designed to have a sweet flavour.

Comment: Sweet flavours include a broad range of flavour choices but they are distinct from fruit flavours and therefore need to described.

This definition was imported from the Addiction Ontology (see https://addictovocab.org/). Comments and suggestions for improvements are welcome using the Qeios review system.

Definitions imported from the Addiction Ontology (AddictO) are what are known as 'ontological definitions'. See this article in Qeios for an explanation https://www.qeios.com/read/YGIF9B.

Ontological definitions can sometimes be hard to read. In those cases we also include an informal definition.

Definitions also often require elaboration to make it clear how they should be used and what they include. In those cases we include a comment.

Also, definitions sometimes require an explanation as to how they came about to help users understand how they relate to alternative definitions. In those cases we include a curator note. 